\title{
ON THE FREE PRODUCT OF TWO GROUPS WITH AN AMALGAMATED SUBGROUP OF FINITE INDEX IN EACH FACTOR'
}

\author{
A. KARRASS AND D. SOLITAR
}

ABstract. Let $G=(A * B ; U)$ where $U$ is finitely generated and of finite index $\neq 1$ in both $A$ and $B$. We prove that $G$ is a finite extension of a free group iff $A$ and $B$ are both finite. In particular, this answers in the negative a question of $W$. Magnus as to whether or not $G$ can be free. Analogous results are obtained for tree products and $\mathrm{HNN}$ groups.

1. Introduction. Let $G=(A * B ; U)$ be a free product with an amalgamated subgroup, where $A, B$ are finitely generated groups. W. Magnus asked whether or not $G$ can ever be a free group if $U$ is of finite index $(\neq 1)$ in both $A$ and in $B$. We prove that $G$ cannot be free in this case, and more generally that $G$ is a finite extension of a free group iff $A$ and $B$ are both finite groups. Moreover, we establish analogous results for a tree product and for an $\mathrm{HNN}$ group of the form

$$
\left\langle t_{1}, \cdots, t_{r}, K \text {; rel } K, t_{1} L_{1} t_{1}^{-1}=M_{1}, \cdots, t_{r}^{-1} L_{r} t_{r}^{-1}=M_{r}\right\rangle
$$

where $K$ is finitely generated and $L_{i}, M_{i}$ are each of finite index in $K$ (see [6] for definitions and notations).

\section{Lemmas.}

LEMMA 1. If $G$ is a free group and $G=(A * B ; U)$ where $U$ is finitely generated and of finite index in both $A$ and $B$, then $A=U$ or $B=U$.

Proof. Since $U$ is finitely generated, $U$ is a free factor of a subgroup $H$ which is of finite index in $G$ (see M. Hall Jr. [3] and [5]). It then follows that

$$
A \cap H=U=B \cap H .
$$

For, $A \cap H$ is a subgroup of $H$ and contains a free factor $U$ of $H$; hence $U$ is a free factor of $A \cap H$. But $U$ is of finite index in $A \cap H$; hence $A \cap H=U$, and similarly $B \cap H=U$.

Received by the editors January 30, 1970 .

AMS 1969 subject classifications. Primary 2052, 2010; Secondary 2054.

Key words and phrases. Amalgamated products, generalized free products, tree products, HNN groups, finite extensions of free groups, free subgroups of finite index, free groups.

1 This research was supported by a grant from the National Research Council of Canada. 
Since $H$ is of finite index in $G, H$ contains a subgroup $H_{1}$, normal and of finite index in $G$. Intersecting the equations (1) with $H_{1}$, we obtain

$$
A \cap H_{1}=U \cap H_{1}=B \cap H_{1} .
$$

Therefore, $U \cap H_{1}$ is a nontrivial normal subgroup of $A$ and of $B$, and hence normal in $G$. Consequently, $U$ is of finite index in $G$, which is impossible unless $A=U$ or $B=U$ (otherwise $(a b)^{n}$ where $a \in A-U$ and $b \in B-U, n=0, \pm 1, \pm 2, \cdots$ determine infinitely many cosets of $U$ in $G$ ).

LeMMA 2. Let $G=\Pi *\left(A_{i} ; U_{j k}=U_{k j}\right)$ be a tree product of groups $A_{i}$ with the subgroups $U_{j k}$ of $A_{j}$ and $U_{k j}$ of $A_{k}$ amalgamated. Suppose $G$ is a free group and each $U_{j k}$ is finitely generated and of finite index in $A_{j}$. Then $G$ equals one of its vertices $A_{n}$ and all the other vertices $A_{i}$ are of finite index in $A_{n}$.

Proof. We first consider the case where $G$ is the tree product of finitely many vertices $A_{1}, \cdots, A_{r}$ and use induction on $r$. Now the finite tree product $G$ has an extremal vertex say $A_{r}$, which is joined to a unique vertex, say $A_{r-1}$. The subgroup of $G$ generated by $A_{1}, \cdots, A_{r-1}$ is just their tree product. Hence by inductive hypothesis, each of these $r-1$ vertices is of finite index in one of them, say $A_{1}$. Then $G=\left(A_{r} * A_{1} ; U_{r, r-1}\right)$, and hence by Lemma 1 , all vertices of $G$ are of finite index in either $A_{1}$ or in $A_{r}$.

Suppose now that $G$ has infinitely many vertices. Let $A_{n}$ be a vertex of minimum rank; moreover, if $A_{n}$ is a cyclic group, we may choose $A_{n}$ to be maximal among the vertices which are cyclic. We show that every other vertex $A_{i}$ of $G$ is of finite index in $A_{n}$. For, $A_{i}$ and $A_{n}$ are contained in a finite subtree of $G$; hence the vertices of this finite subtree are all of finite index in one of them, say $A_{k}$. From the Schreier rank formula, it follows that $A_{k}=A_{n}$.

\section{The theorem for amalgamated products.}

THEOREM 1. Let $G=(A * B ; U)$ where $U$ is finitely generated and of finite index $(\neq 1)$ in both $A$ and $B$. Then $G$ has a free subgroup of finite index iff $A$ and $B$ are both finite groups.

Proof. If $A$ and $B$ are finite, then $G$ has a free subgroup of finite index (see the proof of Theorem 2 in G. Baumslag [1]).

Conversely, suppose $G$ has a free subgroup $H$ of finite index. Then by the subgroup theorem in [6], $H$ is an HNN group of the form 
(3) $\quad H=\left\langle t_{1}, t_{2}, \cdots, t_{r}, S ; \operatorname{rel} S, t_{1} U_{H}^{\delta_{1}} t_{1}^{-1}=U_{H}^{\delta_{1}^{\prime}}, \cdots, t_{r} U_{H}^{\delta_{r}} t_{r}^{-1}=U_{H}^{\delta_{r}^{\prime}}\right\rangle$,

where $S$ is a tree product whose vertices are conjugates of $A$ or $B$ intersected with $H$, and whose amalgamated subgroups are conjugates of $U$ intersected with $H$. Moreover, neighboring vertices may be expressed in the form $A_{H}^{D}, B_{H}^{D}$ with $U_{H}^{D}$ as the subgroup amalgamated between them; also $A_{H}^{\delta_{i}}$ and $B_{H^{i}}^{\delta^{\prime}}$ are among the vertices of $S$.

Since $U$ is finitely generated and of finite index in $A$ and $B$, the subgroup amalgamated between two vertices of $S$ is finitely generated and of finite index in both these vertices. Hence by Lemma $2, S$ is equal to one of its vertices, and each of the vertices of $S$ is of finite index in $S$.

If $N$ is the normal subgroup of $H$ generated by $S$, then $N$ is itself a tree product in which the vertices are the conjugates of $S$ by the freely reduced words in $t_{1}, \cdots, t_{r}$; moreover, neighboring vertices have the form

$$
W t_{i}^{\epsilon}: \overline{t_{i}^{-\epsilon}} W^{-1}, \quad W S W^{-1}
$$

and the subgroup amalgamated between them is

$$
W t_{i}^{e} U_{H}^{\alpha} t_{i}^{e} W^{-1}=W U_{H}^{\beta} W^{-1}
$$

where $\alpha=\delta_{i}, \beta=\delta_{i}^{\prime}$ if $\epsilon=1$, and $\alpha=\delta_{i}^{\prime}, \beta=\delta_{i}$ if $\epsilon=-1$ (see Lemma 2 of [6]). Hence Lemma 2 applies to $N$, and we obtain that $N$ is one of its vertices, so that $N=S$ since $N$ is normal. But then $N$ is a finitely generated normal subgroup of the free group $H$, and so $N=1$, or $N$ is of finite index in $H$ which by (3) implies $N=H$. However, $N \neq H$ because otherwise $H=S$, which by the above is a vertex $A_{H}^{D}$ or $B_{H}^{D}$; thus a conjugate of $H$ is in $A$ or in $B$, contrary to the fact that $A$ and $B$ are of infinite index in $G$. Therefore $N=1$ and both $A$ and $B$ are finite since $A \cap H, B \cap H$ are both in $N$ and of finite index in $A, B$ respectively.

Corollary. Let $G=\Pi *\left(A_{i} ; U_{j k}=U_{k j}\right)$ be a tree product of finitely many groups $A_{i}$ with the subgroups $U_{j k}$ of $A_{j}$ and $U_{k j}$ of $A_{k}$ amalgamated. Suppose each $U_{j k}$ is finitely generated and of finite index in $A_{j}$, and that for some $p, q$ we have $A_{p} \neq U_{p q}$ and $A_{q} \neq U_{q p}$. Then $G$ has a free subgroup of finite index iff each $A_{i}$ is a finite group.

Proof. Suppose first that $G$ has a free subgroup of finite index; then $\left(A_{p} * A_{q} ; U_{p q}=U_{q p}\right)$ also has such. Hence by the above theorem $A_{p}$ must be finite. Moreover, since every vertex of $G$ can be joined to $A_{p}$ by a path each of whose edges is of finite index in the vertices of the edge, each $A_{i}$ must be finite. 
Conversely, suppose each $A_{i}$ is finite. One shows by induction on the number of vertices of $G$ that there is a homomorphism of $G$ onto a finite group which is one-one on each $A_{i}$ and that a normal subgroup having trivial intersection with each $A_{i}$ is free.

\section{An analogous theorem for HNN groups.}

\section{Lemma 3. Let $G$ be an $H N N$ group}

(4) $G=\left\langle t_{1}, \cdots, t_{r}, K\right.$; rel $\left.K, t_{1} L_{1} t_{1}^{-1}=M_{1}, \cdots, t_{r} L_{r} M_{r}^{-1}=M_{r}\right\rangle$.

Then any subgroup $H$ of $G$ which has trivial intersection with each conjugate of $K$ must be a free group.

Proof. Let $X$ be the free group on $x_{1}, x_{2}, \cdots, x_{r}$, and $Y$ the free group on $y_{1}, y_{2}, \cdots, y_{r}$. Then

$$
\begin{aligned}
X * G & =Y * G \\
& =\left((X * K) *(Y * K) ; K * x_{1} L_{1} x_{1}^{-1} * \cdots=K * y_{1} M_{1} y_{1}^{-1} * \cdots\right),
\end{aligned}
$$

where $t_{i}=y_{i}^{-1} x_{i}$ (see Higman, Neumann and Neumann [4] or [6]). We show that the conjugates of $H$ in $X * G$ have trivial intersection with $X * K$. Let $h(\neq 1)$ be an element of $H$, and let $w$ be in $X * G$. When $w h w^{-1}$ is cyclically reduced as an element of $X * G$, an element gh $g^{-1}$ with $g \in G$ results. On the other hand if $w h w^{-1} \in X * K$, then $w h w^{-1}$ when cyclically reduced in $X * G$ must yield an element of $K$, contrary to $H$ having trivial intersection with the conjugates of $K$ in $G$. Similarly, $H$ has trivial intersection with the conjugates of $Y * K$ in $Y * G$. Therefore by a theorem of H. Neumann (see [8] or [6]), $H$ is free.

THEOREM 2. Let $G$ be an HNN group as in (4) where $K$ is finitely generated and each $L_{i}, M_{i}$ is of finite index in $K$. Then $G$ has a free subgroup of finite index iff $K$ is finite. In particular, $G$ cannot be free.

Proof. Suppose first that $K$ is finite. Then $K$ can be embedded in a finite group $\Sigma$ (for example, the group of all permutations on the elements of $K$ ) in such a way that the conjugate of $L_{i}$ by some element $s_{i}$ in $\Sigma$ is $M_{i}$ (see, for example corollary on p. 57 in Carmichael [2], or Philip Hall's proof p. 537 in B. H. Neumann [7]). Map $G$ into $\Sigma$ by sending $K$ identically into itself in $\Sigma$ and mapping $t_{i} \rightarrow s_{i}$. Then $G$ is mapped homomorphically on to a finite group and the kernel is a normal subgroup having trivial intersection with $K$, and hence is free by Lemma 3.

Conversely, suppose that $G$ has a free subgroup of finite index and 
hence a normal free subgroup $H$ of finite index. Suppose $K$ is infinite. We first show that for each $i, L_{i}$ or $M_{i}$ must equal $K$. For otherwise the amalgamated product $\left(K * t_{i} K t_{i}^{-1} ; M_{i}=t_{i} L_{i} t_{i}^{-1}\right)$ is a subgroup of $G$ and hence possesses a free subgroup of finite index, contrary to Theorem 1 . Hence for each $i$, either $L_{i}$ or $M_{i}$ equals $K$. We may therefore assume (replacing $t_{i}$ by $t_{i}^{-1}$ if necessary) that $K=M_{i}$ for some $i$. Let $G_{i}=g p\left(t_{i}, K\right)$ and $H_{i}=G_{i} \cap H$. Then the groups $t_{i}^{j} K t_{i}^{-j} \cap H_{i}$ $=t_{i}^{j}\left(K \cap H_{i}\right) t_{i}^{-j}$ form an ascending chain of free subgroups of the same finite rank in $H_{i}$. Hence for some $j, t_{i}^{j}\left(K \cap H_{i}\right) t_{i}^{-j}=t_{i}^{j+1}\left(K \cap H_{i}\right) t_{i}^{-j-1}$, so that $K \cap H_{i}=t_{i}\left(K \cap H_{i}\right) t_{i}^{-1}$. Therefore $K \cap H_{i}$ is normal in $G_{i}$ and therefore normal in $H_{i}$. But $K \cap H_{i}$ is finitely generated and is therefore of finite index in $H_{i}$ and hence of finite index in $G_{i}$, contrary to $K$ being of infinite index in $G_{i}$. Consequently $K$ must be a finite group.

\section{REFERENCES}

1. G. Baumslag, On the residual finiteness of generalized free products of nilpotent groups, Trans. Amer. Math. Soc. 106 (1963), 193-209. MR 26 \#2489.

2. R. D. Carmichael, An introduction to the theory of groups of finite order, Ginn, Boston, Mass., 1937; reprint Dover, New York, 1956. MR 17, 823.

3. M. Hall Jr., Coset representations in free groups, Trans. Amer. Math. Soc. 67 (1949), 421-432. MR 11, 322.

4. G. Higman, B. H. Neumann and H. Neumann, Embedding theorems for groups, J. London Math. Soc. 24 (1949), 247-254. MR 11, 322.

5. A. Karrass and D. Solitar, On finitely generated subgroups of a free group, Proc. Amer. Math. Soc. 22 (1969), 209-213.

6. - The subgroups of a free product of two groups with an amalgamated subgroup, Trans. Amer. Math. Soc. 150 (1970), 227-255.

7. B. H. Neumann, An essay on free products of groups with amalgamations, Philos. Trans. Roy. Soc. London Ser. A246 (1954), 503-554. MR 16, 10.

8. H. Neumann, (a) Generalized free products with amalgamated subgroups. I, Amer. J. Math. 70 (1948), 590-625. MR 10, 233. (b) II, Amer. J. Math. 71 (1949), 491-540. MR 11, 8.

York University, Toronto, Ontario, Canada 